\title{
Thermal diffusion and quantum chaos in neutral magnetized plasma
}

\author{
Wei $\mathrm{Li}^{1,{ }^{1 *}}$ Shu $\mathrm{Lin}^{2, \dagger}$ and Jiajie $\mathrm{Mei}^{2, *}$ \\ ${ }^{1}$ Department of Physics and Astronomy, National Taiwan University, Taipei 10617, Taiwan \\ ${ }^{2}$ School of Physics and Astronomy, Sun Yat-Sen University, Zhuhai 519082, China
}

(Received 27 May 2019; published 20 August 2019)

\begin{abstract}
We calculate the thermal diffusion constant $D_{T}$ and butterfly velocity $v_{B}$ in neutral magnetized plasma using a holographic magnetic brane background. We find that the thermal diffusion constant satisfies Blake's bound. The constant in the bound $D_{T} 2 \pi T / v_{B}^{2}$ is a decreasing function of magnetic field. It approaches one half in the large-magnetic-field limit. We also find the existence of a special point defined by the Lyapunov exponent and butterfly velocity at which the pole-skipping phenomenon occurs.
\end{abstract}

DOI: $10.1103 /$ PhysRevD.100.046012

\section{INTRODUCTION}

Recently there has been remarkable progress in understanding the connection between transport properties and chaos in strongly coupled many-body systems. On the one hand, the transport of many-body systems is generically governed by a relaxation time scale $\tau$. While the mechanism of relaxation differs between systems, the relaxation time scale is expected to be bounded by the smallest time scale allowed by the uncertainly principle, $\tau \gtrsim \hbar / k_{B} T$ [1]. On the other hand, a similar bound exists for the scrambling rate of the system $\lambda_{L} \leq 2 \pi k_{B} T / \hbar$, with $\lambda_{L}$ being the Lyapunov exponent defined through the out-of-time-order correlator for two Hermitian operators $W$ and $V$ [2-4],

$$
C(t, \vec{x})=-\left\langle[W(t, \vec{x}), V(0,0)]^{2}\right\rangle_{\beta} \sim e^{\lambda_{L}\left(t-t_{*}-\frac{|\vec{x}|}{v_{B}}\right)} .
$$

Here $v_{B}$ is the butterfly velocity and $t_{*}$ is the scrambling time. The bound on the scrambling rate is conjectured to be $2 \pi T$ [5], and the bound is saturated in holographic systems [2] and the Sachdev-Ye-Kitaev (SYK) model [6].

Interestingly, the connections between transport and chaos can be extended. It has been proposed that the diffusion constant, as a proxy of relaxation, satisfies a stronger universal bound: $D \sim \tau \sim \hbar v_{B}^{2} / k_{B} T$ [7]. There has been evidence for this bound for charge diffusion and thermal diffusion in holographic systems [8-19] and SYK models [20-24]. However, the bound for charge diffusion

\footnotetext{
*r07222072@ntu.edu.tw

†linshu8@mail.sysu.edu.cn

\#iajiemei@outlook.com
}

Published by the American Physical Society under the terms of the Creative Commons Attribution 4.0 International license. Further distribution of this work must maintain attribution to the author(s) and the published article's title, journal citation, and DOI. Funded by SCOAP . can be violated with a derivative correction in holographic systems [25] and field theory models [26,27]. The bound for thermal diffusion in general holds; however, see the exceptions in Refs. [28-30]. The nonuniversality/ universality of the bounds can be qualitatively understood in holographic systems: the butterfly velocity is determined by the shock-wave solution, which only probes the nearhorizon region of the gravity background. To determine the diffusion constant, we need to use the Einstein equation. For charge diffusion, $D_{c}=\sigma / \chi$. The conductivity $\sigma$ is also fixed by the IR fixed point. However, the susceptibility $\chi$ is not universal, but rather depends on the full bulk metric. Thus, the bound for charge diffusion can be violated. The situation is different for thermal diffusion, $D_{e}=\kappa / c_{\rho}$. Here the thermal conductivity $\kappa$ is again fixed by the IR fixed point. The specific heat $c_{\rho}$ and $v_{B}$ are determined by the deformation of the fixed point. It has been shown that the same irrelevant deformation enters $c_{\rho}$ and $v_{B}$ [10].

In fact, Ref. [10] described one class of examples where the IR fixed point is $\mathrm{AdS}_{2} \times R^{2}$. In this paper, we present another class where the IR fixed point is given by $B T Z \times R^{2}$. The corresponding holographic model is the magnetic brane solution [31], which interpolates between the IR fixed point to asymptotic $\mathrm{AdS}_{5}$ in the UV. The dual theory is neutral plasma subject to an external magnetic field. The presence of an external magnetic field introduces momentum dissipation to the systems through the Lorentz force, turning the energy density from a propagating mode into a diffusive mode. This is exactly the thermal diffusion we use to test the bound. The validity of the bound for charge diffusion was studied in Ref. [19].

Recently, an intriguing phenomenon of pole-skipping in the correlator of the energy density was studied [32-36]. At a special point $\omega=i \lambda_{L}$ and $k=\frac{i \lambda_{L}}{v_{B}}$ set by chaotic quantities, one component of the Einstein equation is trivially satisfied. This gives rise to an extra independent solution to the metric perturbation, leading to the indeterminacy of the 
energy density correlator at the special point. Moreover, there is a line of poles of the correlator that cross the special point. The pole is skipped at precisely the special point. The existence of the special point and pole-skipping phenomenon shed new light on the connection between quantum chaos and transports. Interestingly, we also confirm the existence of the special point in the magnetic brane background. This provides additional nontrivial evidence for the connection.

The paper is organized as follows. In Sec. II we first present a magnetohydrodynamic derivation of the thermal diffusion mode. Then, we calculate the thermal diffusion constant independently from the magnetic brane background. The results of the two approaches are in agreement with each other. Section III is devoted to the calculation of the butterfly velocity from a shock wave in the magnetic brane background. In Sec. VI we test the bound on the thermal diffusion constant based on the results obtained in the preceding sections. The results are found to be analogous to the case of an incoherent metal. In Sec. V we show the existence of a special point in the magnetic brane background and discuss its implications for magnetohydrodynamics. We conclude and discuss possible extensions in Sec. VI.

\section{THERMAL DIFFUSION IN NEUTRAL MAGNETIZED PLASMA}

\section{A. Thermal diffusion from magnetohydrodynamics}

We first present the derivation of the thermal diffusion constant in the framework of magnetohydrodynamics (MHD) [37,38]. For simplicity we a consider neutral plasma, in which the fluctuations of the energy density and charge density decouple. We are interested in the diffusive mode of the energy density. It arises from the fluctuation of the fluid velocity perpendicular to the magnetic field and energy density (or, equivalently, temperature). The constitutive equation can be expressed as follows:

$$
\begin{aligned}
j_{i} & =\left(E_{i}+\epsilon_{i j k} v_{j} B_{k}\right) \sigma_{\perp}+\partial_{t} P_{i}+\epsilon_{i j k} \partial_{j} M_{k}, \\
T^{00} & =\epsilon, \\
T^{i i} & =p_{\perp}=p_{\|}-M B, \\
T^{0 i} & =\left(\epsilon+p_{\|}\right) v_{i}-\epsilon_{i j k} E_{j} M_{k} .
\end{aligned}
$$

Here the three terms in the current density correspond to the conducting current, polarization current, and magnetization current. We consider plasma subject to a constant external magnetic field and vanishing electric field. It follows that the polarization is induced by the motion of the fluid:

$$
P_{i}=\epsilon_{i j k} v_{j} M_{k} .
$$

The index $i$ in the stress-tensor components in Eq. (2) is transverse to the magnetic field. Without loss of generality, we choose a magnetic field pointing in the $z$ direction and a fluctuating fluid velocity along the $x$ direction. Assuming the plane-wave form $e^{-i \omega t+i k x}$ for the fluctuations, we can write Eq. (2) explicitly:

$$
\begin{aligned}
j_{y} & =-\sigma_{\perp} B v_{x}+i \omega M v_{x}-i k \frac{\partial M}{\partial T} \delta T, \\
\delta T^{00} & =\left(\frac{\partial \epsilon}{\partial T}\right)_{T} \delta T=c_{v} \delta T, \\
\delta T^{x x} & =\delta p-\delta M B=s \delta T-\frac{\partial M}{\partial T} B \delta T, \\
T^{0 x} & =\left(\epsilon+p_{\|}\right) v_{x} .
\end{aligned}
$$

The conservation equations are given by

$$
\begin{aligned}
\partial_{\mu} T^{\mu \nu} & =j_{\lambda} F^{\lambda \nu}, \\
\partial_{\mu} j^{\mu} & =0,
\end{aligned}
$$

with the following nonvanishing components, in our case:

$$
\begin{aligned}
& -i \omega c_{v} \delta T+i k(\epsilon+p) v_{x}=0, \\
& -i \omega(\epsilon+p) v_{x}+i k s \delta T-i \omega M B v_{x}+\sigma_{\perp} B^{2} v_{x}=0 .
\end{aligned}
$$

Equations (4) and (6) are just algebraic equations, which admit two solutions as a dispersion relation. One is the diffusive mode:

$$
\begin{aligned}
\omega & =-\frac{i(\epsilon+p)^{2}}{B^{2} c_{v} T \sigma_{\perp}} k^{2} \\
& =-\frac{i(\epsilon+p)^{2}}{B^{2} T^{2} \frac{\partial s}{\partial T} \sigma_{\perp}} k^{2} .
\end{aligned}
$$

The other is a gapped mode lying beyond the regime of MHD:

$$
\omega=-\frac{i B^{2} \sigma_{\perp}}{p+\epsilon+M B}+\frac{i\left(\epsilon^{2}+p^{2}+2 \epsilon p\right) k^{2}}{B^{2} c_{v} T \sigma_{\perp}} .
$$

Thus, we obtain the thermal diffusion constant as follows:

$$
D_{T}=\frac{(\epsilon+p)^{2}}{B^{2} T^{2} \frac{\partial s}{\partial T} \sigma_{\perp}} .
$$

We will confirm this result with an explicit holographic model calculation.

\section{B. Thermal diffusion from a holographic calculation}

In this section we present a holographic calculation of the thermal diffusion constant in a neutral magnetic brane background [31]. This serves as a confirmation of the result obtained in the previous section based on magnetohydrodynamics. The neutral magnetic brane background is a solution of the Einstein-Maxwell action 


$$
S=\int d^{5} x \sqrt{-g}\left(R+12-\frac{1}{4} F_{M N} F^{M N}\right) .
$$

We have set the overall factor of the action to 1 for simplicity as it does not affect the diffusion constant. We also set the anti-de Sitter radius to 1 . The solution is given as follows:

$$
\begin{aligned}
d s^{2} & =-U d t^{2}+\frac{d r^{2}}{U}+V_{x}\left(\left(d x^{1}\right)^{2}+\left(d x^{2}\right)^{2}\right)+V_{y} d y^{2}, \\
F & =B d x^{1} \wedge d x^{2}
\end{aligned}
$$

Here $U, V_{x}$, and $V_{y}$ are functions of the radial coordinate $r$ only. The constant magnetic field $B$ is along the $y$ direction. The background satisfies the Einstein equation with a backreaction of the gauge field $F$, which leads to anisotropic metric components. The background is dual to a strongly coupled neutral plasma subject to a magnetic field. For plasma at temperature $T$ the background is a black hole, with the warping function $U$ adopting the following expansion near the horizon:

$$
U=4 \pi T\left(r-r_{h}\right)+O\left(\left(r-r_{h}\right)^{2}\right) .
$$

The solution needs to be obtained by numerically integrating the Einstein equation from the horizon to the boundary. A notable feature of this background is that it interpolates between the IR fixed point $B T Z \times R^{2}$ at the horizon and the UV fixed point $\mathrm{AdS}_{5}$ at the boundary.

In general, finding transport coefficients like the diffusion constant amounts to solving linearized bulk equations, which could be complicated for a magnetic brane background. However, it has been shown by Donos, Gauntlett, and Ziogas (DGZ) that for a general class of background, the procedure can be simplified to solving constraint equations near the horizon. The idea of DGZ is to start with the thermodynamic normal mode, which consists of constant perturbations in field theory directions. The normal mode is then promoted to a hydrodynamic mode by including small momenta and frequency. The constraint equations are sufficient to fix the dispersion relation order by order in the small-momenta expansion. We will closely follow this method. For notational simplicity, we shift the horizon to $r=0$ below. To proceed, we need to turn on linear perturbations for the metric and gauge field: $g_{M N} \rightarrow$ $g_{M N}+h_{M N}, A_{M} \rightarrow A_{M}+a_{M}$. Motivated by the MHD analysis, we turn on only a subset of the perturbations. The metric and gauge field perturbations are chosen with the following gauge conditions and satisfy the following expansion near the horizon:

$$
\begin{aligned}
h_{t t} & =e^{-i \omega v}(4 \pi \operatorname{Tr})\left(h_{t t}^{(0)}(x)+O(r)\right), \\
h_{r r} & =e^{-i \omega v} \frac{1}{4 \pi T r}\left(h_{r r}^{(0)}(x)+O(r)\right), \\
h_{i j} & =e^{-i \omega v}\left(h_{i j}^{(0)}(x)+O(r)\right), \\
h_{t r} & =e^{-i \omega v}\left(h_{t r}^{(0)}(x)+O(r)\right), \\
h_{t i} & =e^{-i \omega v}\left(h_{t i}^{(0)}(x)+r h_{t i}^{(1)}(x)+O\left(r^{2}\right)\right), \\
h_{r i} & =e^{-i \omega v} \frac{1}{4 \pi T r}\left(h_{r i}^{(0)}(x)+r h_{r i}^{(1)}(x)+O\left(r^{2}\right)\right), \\
a_{i} & =e^{-i \omega v}\left(a_{i}^{(0)}(x)+O(r)\right),
\end{aligned}
$$

with

$$
\begin{aligned}
2 \pi T\left(h_{t t}^{(0)}(x)+h_{r r}^{0}(x)\right) & =-4 \pi T h_{r t}^{(0)}(x)=p(x), \\
h_{t i}^{(0)}(x) & =h_{r i}^{(0)}(x)=-v_{i}(x),
\end{aligned}
$$

where $i j$ represents the spatial component and $v$ is the ingoing Eddington-Finkelstein coordinate $v=t+\frac{\ln r}{4 \pi T}$. The argument $x$ of the functions with superscript (0) refers to spatial coordinates only.

The master equations are the constraint equations in the Hamiltonian formulation of holographic models [39]. We recall the Arnowitt-Deser-Misner (ADM) decomposition of the metric,

$$
d s^{2}=N^{2} d r^{2}+\gamma_{\mu \nu}\left(d x^{\mu}+N^{\mu} d r\right)\left(d x^{\nu}+N^{\nu} d r\right),
$$

with $\mu=\left(t, x^{1}, x^{2}, y\right)$. In the presence of perturbations, the functions in the ADM form are given by

$N=U^{-1 / 2}\left(1+\frac{1}{2} U h_{r r}\right), \quad N_{t}=-\frac{1}{U} h_{r t}, \quad N_{i}=h_{r i}$.

In the Hamiltonian formulation, we define the stress tensor and charge current on each $r$ hypersurface as

$$
\begin{aligned}
\pi^{\mu \nu} & =K h^{\mu \nu}-K^{\mu \nu}, \\
J^{\mu} & =-N^{-1}\left(F_{r}^{\mu}-N_{\nu} F^{\nu \mu}\right) .
\end{aligned}
$$

Here we define these without $\sqrt{-\gamma}$ as a tensor and vector with respect to the induced metric $\gamma_{\mu \nu}$. The extrinsic curvature is defined as $K_{\mu \nu}=\frac{1}{2}\left(\dot{\gamma}_{\mu \nu}-\nabla_{\mu} N_{\nu}-\nabla_{\nu} N_{\mu}\right)$. With these definitions, the constraint equations are energy-momentum conservation and charge conservation, which can be written as

$$
\begin{aligned}
H_{\mu} & \equiv 2 \nabla_{\nu} \pi_{\mu}^{\nu}-F_{\mu \nu} J^{\nu}=0, \\
C & =\nabla_{\mu} J^{\mu}=0 .
\end{aligned}
$$


Plugging the perturbations (13) into Eq. (18) and expanding terms to leading order in $r$, we end up with the following equations:

$$
\begin{aligned}
& \partial_{i} Q_{(0)}^{i}-i \omega(2 \pi T) \sqrt{-g_{(0)}} g_{(0)}^{i j} h_{i j}^{(0)}=0, \\
& -2 \partial^{j} \partial_{[j} v_{i]}+\left(1+\frac{i \omega}{4 \pi T}\right) \partial_{i} p+i \omega\left(-h_{t i}^{(1)}+\partial_{i} h_{t r}^{(0)}-g_{i k}^{(1)} v^{k}+\partial^{k} h_{k i}^{(0)}+\frac{i \omega}{4 \pi T}\left(h_{t i}^{(1)}-h_{r i}^{(1)}\right)\right) \\
& +F_{(0)}^{i k}\left(-g_{(0)}^{l j} F_{j k}^{(0)} v_{l}-i \omega a_{k}^{(0)}\right)=0, \\
& i \omega \partial_{i}\left(g_{(0)}^{i j} a_{j}^{(0)}\right)=0,
\end{aligned}
$$

with $Q_{(0)}^{i}=4 \pi T \sqrt{-g_{(0)}} v^{i}$ being the thermal current. The three equations correspond to energy conservation, momentum conservation, and charge conservation, respectively.

Let us begin with the thermodynamic normal mode. This is obtained by an infinitesimally constant shift of temperature $\delta T$ of the background solution. In the neutral background we consider here, the thermal current decouples from the charge current, so that we do not need to consider a shift in the chemical potential for the normal mode. The temperature shift is by construction a normal mode. The corresponding nonvanishing perturbations are given by

$h_{t t}^{T H}=-4 \pi r \delta T, \quad h_{r r}^{T H}=-\frac{\delta T}{4 \pi T^{2} r}, \quad h_{i j}^{T H}=\frac{\partial g_{i j}^{(0)}}{\partial T} \delta T$.

However, this normal mode does not satisfy the gauge condition (14). We can satisfy the gauge condition using the coordinate transformation $t \rightarrow t+\frac{\delta T}{T} g(r)$. With a properly chosen function $g(r)=\ln r /(4 \pi T)+g^{(1)} r$ near the horizon, the normal mode transforms to the following form near the horizon:

$$
\begin{aligned}
& h_{t t}^{R T}=-4 \pi r \delta T, \quad h_{r r}^{R T}=-\frac{\delta T}{4 \pi T^{2} r}, \\
& h_{t r}^{R T}=-\frac{\delta T}{T}, \quad h_{i j}=\frac{\partial g_{i j}^{(0)}}{\partial T} \delta T .
\end{aligned}
$$

We can read off the coefficients $h_{t t}^{(0)}=h_{r r}^{(0)}=h_{t r}^{(0)}=-\frac{\delta T}{T}$ and $p=4 \pi T$ when comparing with Eq. (13). This clearly solves Eq. (19) with $\omega=0$.

Now the crucial step is to promote the temperature to be a slowly varying function of $x: \delta T(x)=e^{i \epsilon k_{i} x^{i}} \delta T$, with $\epsilon$ being a bookkeeping parameter. The promoted function then no longer satisfies the bulk equation, but rather needs to be corrected with $\omega$ and $k$ dependence. The correction can be done order by order in $\epsilon$. The corrected solution assumes the following form [40]:

$$
\begin{aligned}
h_{\mu \nu} & =e^{-i \omega v+i \epsilon k_{i} x^{i}}\left(h_{\mu \nu}^{R T}(x)+\epsilon h_{[1] \mu \nu}(x)+\epsilon^{2} h_{[2] \mu \nu}(x)+\cdots\right), \\
a_{\mu} & =e^{-i \omega v+i \epsilon k_{i} x^{x}}\left(\epsilon a_{[1] \mu \nu}(x)+\epsilon^{2} a_{[2] \mu \nu}(x)+\cdots\right) .
\end{aligned}
$$

Written explicitly in terms of the fields in Eq. (13) and the constant $\delta T$, we have

$$
\begin{aligned}
h_{i j}^{(0)} & =e^{i \epsilon k_{i} x^{i}}\left(\frac{\partial g_{i j}^{(0)}}{\partial T} \delta T+h_{[1] i j}^{0}+\cdots\right), \\
v_{i} & =e^{i \epsilon k_{i} x^{i}}\left(\epsilon v_{[1] i}+\epsilon^{2} v_{[2] i}+\cdots\right), \\
p & =e^{i \epsilon k_{i} x^{i}}\left(4 \pi \delta T+\epsilon p_{[1]}+\cdots\right), \\
\omega & =\epsilon \omega_{[1]}+\epsilon^{2} \omega_{[2]}+\cdots .
\end{aligned}
$$

Note that except for $p$ and $h_{i j}$, which are nonvanishing at $O\left(\epsilon^{0}\right)$ in the thermodynamic normal mode (21), all of the quantities begin with $O(\epsilon)$. The power counting is also consistent with our MHD analysis, where $\delta T / v_{x} \sim k / \omega \sim 1 / k \sim 1 / \epsilon$. It holds for $k \perp v$, which we assume below. For $k \| v$, a different power counting needs to be assumed. Now we can plug Eq. (23) into Eq. (19) and solve it order by order in $\epsilon$ to determine the dispersion relation $\omega(k)$. In fact, we only need the first equation in Eq. (19), which is from energy conservation. Furthermore, since our background is homogeneous, the coefficients of the expansion in Eq. (23) are constants, and thus we would have algebraic equations. Following DGZ, we write the energy conservation equation as the conservation of thermal current. At $O(\epsilon)$, it gives

$$
i \omega_{[1]} g_{(0)}^{i j} \frac{\partial h_{i j}^{(0)} 0}{\partial T}=0,
$$

and thus $\omega_{[1]}=0$. At $O\left(\epsilon^{2}\right)$, noting that $T(x)=T+$ $e^{i \epsilon k_{i} x^{i}} \delta T$, we obtain

$$
2 i k_{i} v_{[1]}^{i}=i \omega_{[2]} g_{(0)}^{i j} \frac{\partial h_{i j}^{(0)}}{\partial T}
$$

To solve for $\omega_{[2]}$, we need to know $v_{[1]}^{i}$. This can be obtained in the following way. To first order in $\epsilon, v_{[1]}^{i}$ simply gives 
the horizon thermal current $Q_{(0)}^{i}$, which can be expressed as a response to the temperature gradient:

$4 \pi T \sqrt{-g_{(0)}} \epsilon v_{[1]}^{i}=Q_{(0)}^{i}=-\kappa_{H}^{i j} \partial_{j}(T(x))=-i \epsilon \kappa_{H}^{i j} k_{j} \delta T$,

where $\kappa_{H}$ is the horizon thermal conductivity. Plugging this into Eq. (25), we obtain the dispersion relation

$$
\omega_{[2]}=\frac{-i k_{i} k_{j} \kappa_{H}^{i j}}{c_{v}},
$$

where we have used $c_{v}=T \frac{\partial s}{\partial T}=\frac{4 \pi T \partial \sqrt{-g_{(0)}}}{\partial T}$. Note that we have $k \perp v$ and also $k \perp B$. From this, we extract a diffusion constant $D_{T}=\frac{\kappa}{c_{v}}$. Here $\kappa$ corresponds to the transverse thermal conductivity and it has been shown that it does not flow along the radial direction [41]; thus, we omit the subscript $H$.

Finally, we show that Eq. (27) actually matches our result from magnetohydrodynamics. First, we recall the Kubo formula derived from MHD [37],

$$
\lim _{\omega \rightarrow 0} \frac{1}{\omega} \operatorname{Im} G_{T_{t x} T_{t x}}=\frac{w^{2}}{\sigma_{\perp} B^{2}},
$$

and the definition of thermal conductivity from the thermal current,

$\kappa=\frac{1}{T} \lim _{\omega \rightarrow 0} \frac{1}{\omega} \operatorname{Im} G_{Q_{x} Q_{x}}=\frac{1}{T} \lim _{\omega \rightarrow 0} \frac{1}{\omega} \operatorname{Im} G_{T_{t x} T_{t x}}=\frac{1}{T} \frac{w^{2}}{\sigma_{\perp} B^{2}}$.

where $w=\varepsilon+p=T s$ by the first law of thermodynamics. Therefore, the thermal diffusion constant is

$$
D_{T}=\frac{\kappa}{c_{v}}=\frac{w^{2}}{\sigma_{\perp} B^{2} \frac{\partial s}{\partial T} T^{2}} .
$$

This is in perfect agreement with our MHD result in the previous section.

We will relate the thermal diffusion constant to chaotic quantities in the next section. It is instructive to express $D_{T}=\kappa / c_{v}$ in terms of metric functions. Using $s=$ $4 \pi \sqrt{V_{x}^{2}\left(r_{h}\right) V_{y}\left(r_{h}\right)}$ and $\sigma_{\perp}=\sqrt{V_{y}\left(r_{h}\right)}$ [42], we can express $\kappa$ solely with metric at the IR fixed point. Since $c_{v}=\frac{\partial s}{\partial T}, D_{T}$ can be expressed as

$D_{T}=\left.8 \pi \frac{V_{x}(r)}{B^{2}}\left(\frac{2}{V_{x}(r)} \frac{\partial V_{x}(r)}{\partial T}+\frac{1}{V_{y}(r)} \frac{\partial V_{y}(r)}{\partial T}\right)\right|_{r=r_{h}}$.

\section{SHOCK WAVE AND QUANTUM CHAOS}

The chaotic quantities including the Lyapunov exponent and butterfly velocity can be calculated using a shock-wave solution in a magnetic brane background [3]. Since a magnetic brane is anisotropic, we adopt the method used in Ref. [13]. In Kruskal coordinates the anisotropic metric is given by

$$
\begin{aligned}
\mathrm{d} s^{2}= & A(u v) \mathrm{d} u \mathrm{~d} v+V_{x}(u v)\left(\left(\mathrm{d} x^{1}\right)^{2}+\left(\mathrm{d} x^{2}\right)^{2}\right) \\
& +V_{y}(u v) \mathrm{d} y^{2},
\end{aligned}
$$

where $u v=-e^{U^{\prime}\left(r_{h}\right) r_{*}(r)}$ and $u / v=-e^{-U^{\prime}\left(r_{h}\right) t}$, with $r_{*}$ being the tortoise coordinate defined by $\mathrm{d} r_{*}=\mathrm{d} r / U(r)$. In addition, $A(u v)=\frac{4 U(r)}{u v U^{\prime}\left(r_{h}\right)^{2}}$. Note that in this coordinate system the horizon is at $u=v=0, r=r_{h}$.

Consider an operator insertion at time $t$ into the thermal field double state; this is dual to a particle injection into the background in the gravitational description. The boundary time evolution corresponds to a boost of the particle energy in the Kruskal coordinates. In particular, a particle with energy $E_{0}$ will have energy $E_{0} e^{\frac{2 \pi}{\beta} t}$ in local time. When $e^{\frac{2 \pi}{\beta} t} \sim O\left(N^{2}\right)$, the backreaction of the particle on the background geometry can no longer be neglected. This fixes the scrambling time $t_{*} \sim \beta \log N^{2}$. The backreacted geometry is given by a shock wave localized at the horizon in the original background. In Kruskal coordinates the resulting stress tensor of the particle is given by

$$
\left(\delta T_{u u}\right)_{\text {particle }} \sim E_{0} e^{\frac{2 \pi}{\beta} t} \delta(u) \delta(\vec{x}) .
$$

The corresponding shock-wave background is given by

$$
\begin{aligned}
\mathrm{d} s^{2}= & A(u v) \mathrm{d} u \mathrm{~d} v-A(u v) \delta(u) h(\vec{x}) \mathrm{d} u^{2} \\
& +V_{x}(u v)\left(\left(\mathrm{d} x^{1}\right)^{2}+\left(\mathrm{d} x^{2}\right)^{2}\right)+V_{y}(u v) \mathrm{d} y^{2} .
\end{aligned}
$$

The perturbed Einstein equation can be written as

$$
\delta G_{u u}=\left(\delta T_{u u}\right)_{\text {particle }},
$$

which leads to the following equation for $h(\vec{x})$ :

$$
\begin{aligned}
& \left(\frac{1}{V_{x}\left(r_{h}\right)} \partial_{x_{1}}^{2}+\frac{1}{V_{x}\left(r_{h}\right)} \partial_{x_{2}}^{2}+\frac{1}{V_{y}\left(r_{h}\right)} \partial_{y}^{2}-m^{2}\right) h(\vec{x}) \\
& \quad \sim \frac{16 \pi G_{N}}{A(0)} E_{0} e^{\frac{2 \pi}{\beta} t} \delta(\vec{x})
\end{aligned}
$$

with the effective mass $m^{2}$ given by

$$
\begin{aligned}
m^{2} & =\left.\frac{1}{A(u v)}\left(\frac{2 V_{x}^{\prime}(u v)}{V_{x}(u v)}+\frac{V_{y}^{\prime}(u v)}{V_{y}(u v)}\right)\right|_{u=0} \\
& =\pi T\left(\frac{2 V_{x}^{\prime}\left(r_{h}\right) V_{y}\left(r_{h}\right)+V_{y}^{\prime}\left(r_{h}\right) V_{x}\left(r_{h}\right)}{V_{x}\left(r_{h}\right) V_{y}\left(r_{h}\right)}\right) .
\end{aligned}
$$


Equation (36) is a type of Poisson equation, which can be solved as

$$
h\left(x_{1}, x_{2}, y\right) \sim \frac{E_{0} e^{\frac{2 \pi}{\beta}\left(t-t_{*}\right)-m|x|}}{|x|},
$$

where $|x|$ is given by

$$
|x|=\sqrt{V_{x}\left(r_{h}\right) x_{1}^{2}+V_{x}\left(r_{h}\right) x_{2}^{2}+V_{y}\left(r_{h}\right) y^{2}} .
$$

The exponent in Eq. (38) gives both the Lyapunov exponent and butterfly velocity. The Lyapunov exponent $\lambda_{L}=2 \pi T$ saturates the Maldacena-Shenker-Stanford bound, as expected. The butterfly velocity is anisotropic. To calculate it in the $x_{1}$ direction, we can set $x_{2}=y=0$ to get

$$
v_{x 1}^{2}=\frac{4 \pi^{2} T^{2}}{V_{x}\left(r_{h}\right) m^{2}} .
$$

Similarly, in different directions we get

$$
v_{x 1}^{2}=v_{x 2}^{2}=\frac{4 \pi^{2} T^{2}}{V_{x}\left(r_{h}\right) m^{2}}, \quad v_{y}^{2}=\frac{4 \pi^{2} T^{2}}{V_{y}\left(r_{h}\right) m^{2}} .
$$

\section{THERMAL DIFFUSION BOUND}

Now we are ready to verify the bound on the thermal diffusion constant from Eqs. (31) and (41). Note that the latter depends only on the horizon metric, but the former also depends on the variation of the horizon metric with temperature, which needs to be obtained from the numerical solution of the magnetic brane background. To test the bound, we calculate the ratio $\lambda_{L} D_{T} / v_{B}^{2}$ :

$$
\begin{aligned}
\lambda_{L} D_{T} / v_{B}^{2}= & 4 \pi \frac{V_{x}(r)^{2}}{B^{2}}\left(\frac{2 V_{x}^{\prime}(r)}{V_{x}(r)}+\frac{V_{y}^{\prime}(r)}{V_{y}(r)}\right) \\
& \times\left.\left(\frac{2 \partial V_{x}(r) / \partial T}{V_{x}(r)}+\frac{\partial V_{y}(r) / \partial T}{V_{y}(r)}\right)^{-1}\right|_{r=r_{h}} .
\end{aligned}
$$

Here we take the transverse butterfly velocity. We show the dependence of the ratio on the external magnetic field in Fig. 1. It is a monotonically decreasing function with an asymptotic value of $1 / 2$. In fact, the asymptotic value can be confirmed analytically from the large- $B$ limit. In this case, the background is simply given by $B T Z \times R^{2}$ from dimensional reduction [31]. With our standard normalization for the electromagnetic field, the explicit metric is given by

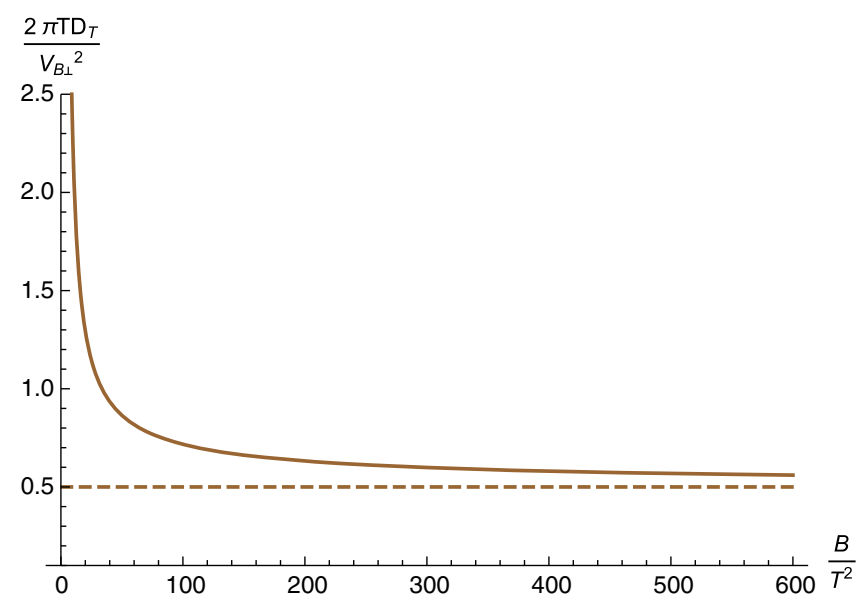

FIG. 1. The ratio $\lambda_{L} D_{T} / v_{B}^{2}$ as a function of $B / T^{2}$. It is a monotonically decreasing function with an asymptotic value of $1 / 2$. The asymptotic value can be obtained from the dimensionally reduced metric $B T Z \times R^{2}$ in the large- $B$ limit.

$$
\begin{aligned}
d s^{2}= & -3\left(r^{2}-r_{h}^{2}\right) d t^{2}+\frac{d r^{2}}{3\left(r^{2}-r_{h}^{2}\right)} \\
& +\frac{B}{2 \sqrt{3}}\left(\left(d x^{1}\right)^{2}+\left(d x^{2}\right)^{2}\right)+3 r^{2} d y^{2} .
\end{aligned}
$$

Noting that $r_{h}=2 \pi T / 3$, we can easily verify that $\lambda_{L} D_{T} / v_{B}^{2}=1 / 2$.

It is instructive to compare the case of a magnetized plasma with the case of an incoherent metal [8]. The external magnetic field in our case exerts a Lorentz force on the plasma charge carriers, introducing momentum dissipation, just like for the lattice in a metal. The large- $B$ limit corresponds to the incoherent metal limit. However, the asymptotic value of the ratio $\lambda_{L} D_{T} / v_{B}^{2}=1$ in the incoherent metal case differs from ours. In a sense, the incoherent metal case is closer to a four-dimensional (4D) magnetic black hole, which is dual to a $2+1 D$ plasma subject to an external magnetic field. In the Appendix we verify that in the $4 \mathrm{D}$ magnetic black hole case the ratio $\lambda_{L} D_{T} / v_{B}^{2}$ approaches 1 from above. In fact, the 4D magnetic black hole has the same IR fixed point $\left(\mathrm{AdS}_{2} \times R^{2}\right)$ as the incoherent metal. In contrast, the five-dimensional magnetic black hole (brane) has an anisotropic IR fixed point $\left(B T Z \times R^{2}\right)$. Interestingly, if we consider a black hole in $d+1$ dimensions with a maximally symmetric magnetic field (as in Ref. [31]), the IR fixed point is $\mathrm{AdS}_{2} \times R^{d-1}$ for even $d$ and $B T Z \times R^{d-2}$ for odd $d$. In the Appendix, we determine that the corresponding asymptotic ratios are 1 and $1 / 2$, respectively. The difference between the two cases is that the background is isotropic/anisotropic for $d$ even/ odd, respectively. It is also worth noting that the holographic model for a metal with hyperscaling violation gives the asymptotic value $\lambda_{L} D_{T} / v_{B}^{2}=\frac{z}{2 z-2}$, with $z$ being the dynamical critical exponent $[8,13]$. It approaches $1 / 2$ as $z \rightarrow \infty$. 


\section{A SPECIAL POINT AND POLE-SKIPPING PHENOMENON}

It was recently found that there is a special point in the energy density correlator. The special point is set entirely by chaotic quantities,

$$
\omega=i \lambda_{L}, \quad k=i \frac{\lambda_{L}}{v_{B}} .
$$

Note that the frequency and momentum are purely imaginary. At the special point, the energy density correlator becomes undetermined, but it depends on how the special point is approached [34]. The indeterminacy of the correlator is related to a special property of the Einstein equation in holographic systems: the $v v$ component of the Einstein equation is trivially satisfied, with $v$ being the EddingtonFinkelstein coordinate. In particular, it has been shown that generically the Einstein equation near the horizon has more than one solution [34], which leads to the indeterminacy of the energy density correlator. We will show that this is also true for a magnetic brane solution, in which the special point is realized in a somewhat nontrivial way.

We start by writing the magnetic brane solution in Eddington-Finkelstein coordinates,

$$
\begin{aligned}
d s^{2}= & -U(r) d v^{2}+2 d v d r+V_{x}(r)\left(\left(d x^{1}\right)^{2}+\left(d x^{2}\right)^{2}\right) \\
& +V_{y}(r) d y^{2} .
\end{aligned}
$$

Near the horizon, the metric functions have the following expansions:

$$
\begin{gathered}
U=4 \pi T\left(r-r_{h}\right)+u_{2}\left(r-r_{h}\right)^{2}+\cdots, \\
V_{x}=1+v x_{1}\left(r-r_{h}\right)+\cdots, \\
V_{y}=1+v y_{1}\left(r-r_{h}\right)+\cdots .
\end{gathered}
$$

Note that the leading terms in the expansions of $U$ and $V_{x}\left(V_{y}\right)$ are $4 \pi T$ and 1 , respectively. The former is determined by the magnetic brane temperature. The latter is arbitrary. It can be realized by rescaling the $x_{1}, x_{2}$, and $y$ coordinates [31]. The rescaling also changes the value of the magnetic field, which we take as $b$. It is easy to obtain the higher-order coefficients of the expansion as

$u_{2}=\frac{5 b^{2}-24}{12}, \quad v x_{1}=\frac{-b^{2}+12}{6 \pi T}, \quad v y_{1}=\frac{b^{2}+24}{12 \pi T}$.

Now that we turn on the following fluctuations: $h_{v v}\left(v, x_{1}, r\right), h_{v r}\left(v, x_{1}, r\right), h_{r r}\left(v, x_{1}, r\right), h_{v x 1}\left(v, x_{1}, r\right)$, and $h_{x 1 \times 1}\left(v, x_{1}, r\right)$. The fluctuations assume the following expansion near the horizon:

$$
h_{v v}=e^{i \omega v+i k x_{1}}\left(h_{v v 0}+h_{v v 1}\left(r-r_{h}\right)+\cdots\right),
$$

and similarly for the other components. The $v v$ component of the Einstein equation can be expanded near the horizon using Eqs. (46) and (49) with the leading coefficient

$$
\begin{aligned}
& \left(\frac{k^{2}}{2}+\frac{i \omega\left(b^{2}-24\right)}{16 \pi T}\right) h_{v v 0}+\frac{2 \omega^{2}-4 i \pi T \omega}{4} h_{x x 0} \\
& +\frac{4 k \omega-8 i \pi T k}{4} h_{v x 0}=0 .
\end{aligned}
$$

We find that there is a special point at

$$
\omega=2 i \pi T, \quad k=i \frac{\sqrt{24-b^{2}}}{2} .
$$

On the other hand, we can express the butterfly velocity in terms of the metric (46). We can simply apply Eq. (41) and use Eq. (48) to obtain

$$
v_{x}=\frac{4 \pi T}{\sqrt{b^{2}-24}} .
$$

We can readily verify the special point (44) by noting that $\lambda_{L}=2 \pi T$. Note that both Eqs. (51) and (52) are subject to a rescaling to coordinates with proper normalization at the boundary. Nevertheless, the rescaling of the $x_{1}$ coordinate acts on $k$ and $v_{x}$ in the opposite way, leaving the form of Eq. (44) invariant. The rescaling in other coordinates does not lead to any change. Therefore, we confirm the existence of the special point (44) in the magnetic brane background as well, independent of the strength of the magnetic field.

Remarkably, the special point is found to lie on the branch of the quasinormal mode whose low-momenta limit corresponds to the hydrodynamic diffusive mode [34]. Note that for purely imaginary momenta, the diffusive mode becomes unstable. In particular, the hydrodynamic description of the unstable mode seems more and more accurate in the incoherent metal limit [34] in the sense that the ratio $D \lambda_{L} / v_{B}^{2}$ approaches one. In other words, the chaotic diffusion constant defined by the special point tends to the hydrodynamic diffusion constant at low energy:

$$
D=\left.\frac{i \lambda_{L}}{i\left(i \lambda_{L} / v_{B}\right)^{2}} \rightarrow \frac{\omega}{i k^{2}}\right|_{k \rightarrow 0} .
$$

In our magnetized plasma case, we find a slightly different result. The ratio $D \lambda_{L} / v_{x}^{2}$ approaches one half in the limit of a large magnetic field. This limit is analogous to the incoherent metal limit as both correspond to large momentum dissipation. It seems that in our case the chaotic diffusion constant and hydrodynamic diffusion constant agree at a finite value of the magnetic field. Our numerical result in Fig. 2 indicates that this occurs at $B / T^{2}=33.67$. It would be interesting to explore the properties of MHD near this value of the magnetic field. However, this interpretation comes with certain caveats. We have not confirmed 


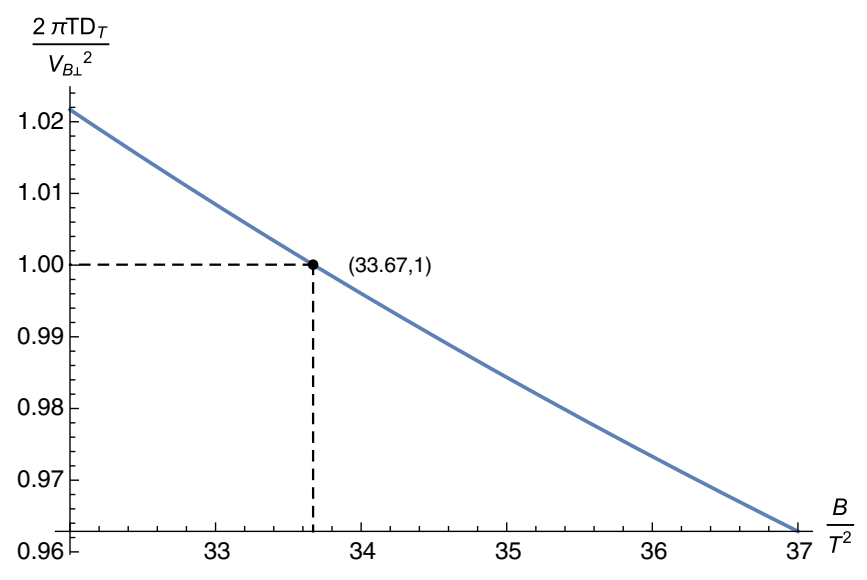

FIG. 2. The ratio $\lambda_{L} D_{T} / v_{B}^{2}$ approaches 1 when $B / T^{2} \approx 33.67$. We expect that near this region hydrodynamics is a good approximation.

that the special point lies on the branch of the quasinormal mode whose low-momenta limit corresponds to the hydrodynamic diffusive mode. Also, we have not considered high-order corrections to the diffusive mode. We leave these for future studies.

\section{SUMMARY AND OUTLOOK}

We calculated the transverse thermal diffusion constant $D_{T}$ in a magnetic brane background. We also calculated the Lyapunov exponent $\lambda_{L}$ and butterfly velocity $v_{B}$ in the same background. We found that Blake's bound on the thermal diffusion constant holds in a magnetic brane background as well. In particular, the ratio $D_{T} \lambda_{L} / v_{B \perp}^{2}$ is a monotonically decreasing function of $B / T^{2}$ with an asymptotic value of $1 / 2$.

We also found the existence of a special point $\omega=i \lambda_{L}$ and $k=i \lambda_{L} / v_{x}$. At this point, the energy density correlator becomes undetermined, similar to the case of an incoherent metal. There is one interesting difference with the incoherent metal case: in the limit of strong momentum dissipation, the ratio $D_{T} \lambda_{L} / v_{B \perp}^{2}$ tends to $1 / 2$ in our case, while it approaches 1 in the incoherent metal case. The deviation from 1 in our case seems to be due to the anisotropy of the background. A similar deviation of the asymptotic value of the ratio from 1 is also seen in holographic models with hyperscaling violation. It would be interesting to explore further the magnetohydrodynamics at large $B$ and shock waves in the $B T Z \times R^{2}$ background where analytic calculations are possible.

The present analysis can be readily generalized to the charged magnetic brane background dual to a charged magnetized plasma, in which mixing between charge diffusion and thermal diffusion is expected [1]. It would offer a further nontrivial test of the intriguing connection between low-energy transport and chaos.

While this work focused on plasma subject to external magnetic field, it would also be very interesting to study the case of plasma with a dynamical electromagnetic field. In fact, holographic models corresponding to a dynamical electromagnetic field were constructed in Refs. [43,44] along the lines of a dual formulation of MHD [38]. This is expected to shed more light on the role of magnetic fields in the connection between transport and chaos. We leave this for a future study.

\section{ACKNOWLEDGMENTS}

It is a pleasure to thank Xian Gao, Xian-hui Ge, Yan Liu, Jia-rui Sun, and especially Wei-jia Li for useful discussions. S. L. would like to thank the University of Science and Technology of China for hospitality during the final stage of this work. S. L. is supported by the One Thousand Talent Program for Young Scholars and NSFC under Grants No. 11675274 and 11735007 . J. J. M. would like to thank Sun Yat-Sen University for hospitality while part of this work was completed.

\section{APPENDIX: DIFFUSION BOUND IN 2+1D AND HIGHER DIMENSIONS}

Here we consider the energy diffusion in a $2+1 D$ plasma, which is dual to an $\mathrm{AdS}_{4}$ black hole with an external magnetic field.

$$
S=\frac{1}{16 \pi G_{4}} \int \mathrm{d}^{4} x \sqrt{-g}\left[R+\frac{6}{L^{2}}-\frac{1}{4} F^{\mu \nu} F_{\mu \nu}\right] .
$$

The neutral magnetized plasma solution is given by [31]

$$
\begin{aligned}
\mathrm{d} s^{2} & =-U(r) \mathrm{d} t^{2}+\frac{\mathrm{d} r^{2}}{U(r)}+r^{2} \mathrm{~d} x^{2}+r^{2} \mathrm{~d} y^{2}, \\
A & =B x \mathrm{~d} y, \quad U(r)=r^{2}+\frac{B^{2}}{4 r^{2}}+\frac{1}{r}\left(\frac{-B^{2}-4 r_{h}^{4}}{4 r_{h}}\right) .
\end{aligned}
$$

Here we set $16 \pi G_{4}=1$ and $L=1$. So the thermal conductivity and the specific heat are [13]

$\kappa=\frac{4 \pi s T r_{h}^{2}}{B^{2}}, \quad c_{v}=T\left(\frac{\partial s}{\partial T}\right)_{B}=8 \pi T r_{h}\left(\frac{\partial r_{h}}{\partial T}\right)_{B}$.

Using the definition of the black hole temperature, we get the horizon radius as a root of the equation

$$
-\frac{B^{2}+2 r_{h}^{2}\left(-6 r_{h}^{2}+8 \pi r_{h} T\right)}{16 \pi r_{h}^{3}}=0,
$$

from which we further get

$$
\left(\frac{\partial r_{h}}{\partial T}\right)_{B}=\frac{16 \pi r_{h}}{12 r_{h}+3 B^{2}} .
$$




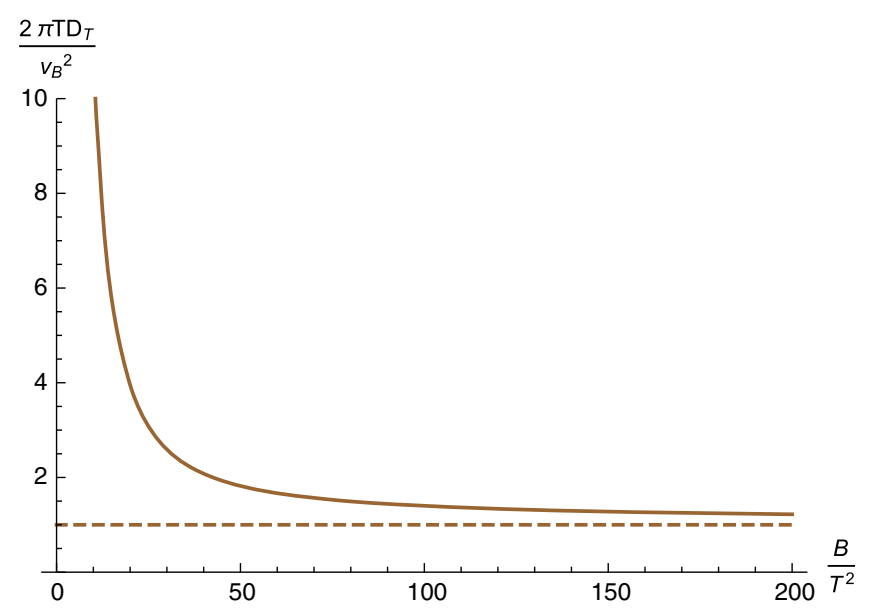

FIG. 3. The ratio $\lambda_{L} D_{T} / v_{B}^{2}$ as a function of $B / T^{2}$. The dashed line represents a constant value of 1 . The bound is a monotonically decreasing function with an asymptotic value of 1 .

So the thermal diffusion constant $D_{T}$ is

$$
D_{T}=\frac{3}{8 r_{h}}\left(\frac{4 r_{h}^{4}}{B^{2}}+1\right)
$$

The butterfly velocity in this case is $v_{B}^{2}=\pi T / r_{h}^{2}$, so the ratio of the diffusion bound is given by

$$
\frac{2 \pi T D_{T}}{v_{B}^{2}}=\frac{3 r_{h}^{4}}{B^{2}}+\frac{3}{4}
$$

When $B / T^{2} \gg 1, r_{h}$ approaches a constant $r_{h}^{2} \rightarrow r_{0}^{2}=\frac{B}{\sqrt{12}}$, and the near-horizon geometry will become $\mathrm{AdS}_{2} \times R^{2}$,

$\mathrm{d} s^{2}=-6\left(r-r_{0}\right)^{2} \mathrm{~d} t^{2}+\frac{\mathrm{d} r^{2}}{6\left(r-r_{0}\right)^{2}}+\frac{B}{\sqrt{12}} \mathrm{~d} x^{2}+\frac{B}{\sqrt{12}} \mathrm{~d} y^{2}$.

So the bound ratio is $2 \pi T D_{T} / v_{B}^{2}=1$. In Fig. 3 we show the numerical result of the bound when $B / T^{2}$ is finite, and we can see that the asymptotic value matches the analysis of the near-horizon geometry $\mathrm{AdS}_{2} \times R^{2}$ in the large- $B$ limit.

For a higher-dimensional magnetic brane, we consider only the limiting case of a large and maximally symmetric magnetic field [31]. When $d$ is even, it has an IR fixed point solution, which is just $B T Z \times R^{d-2}$,

$$
\begin{aligned}
d s^{2}= & -(d-1)\left(r^{2}-r_{h}^{2}\right) d t^{2}+\frac{1}{(d-1)\left(r^{2}-r_{h}^{2}\right)} d r^{2} \\
& +(d-1) r^{2} d y^{2}+\frac{B}{2 \sqrt{d-1}}\left(\left(d x^{1}\right)^{2}+\cdots\left(d x^{d-2}\right)^{2}\right),
\end{aligned}
$$

with

$$
F_{12}=F_{34}=\cdots=B \rightarrow \infty, \quad F_{i y}=0 .
$$

The entropy is given by

$$
\begin{aligned}
s & =4 \pi \sqrt{g_{x_{1} x_{1}}\left(r_{h}\right) \cdots g_{x_{d-2} x_{d-2}}\left(r_{h}\right) g_{y y}\left(r_{h}\right)} \\
& =8 \pi^{2} T\left(\frac{B}{2}\right)^{\frac{d}{2}-1}(d-1)^{-\frac{d}{4}} .
\end{aligned}
$$

The transverse conductivity is

$$
\begin{aligned}
\sigma_{\perp} & =\sqrt{g_{x_{1} x_{1}}\left(r_{h}\right) \cdots g_{x_{d-2} x_{d-2}}\left(r_{h}\right) g_{y y}\left(r_{h}\right)} g^{x_{1} x_{1}}\left(r_{h}\right) \\
& =2 \pi T\left(\frac{B}{2}\right)^{\frac{d}{2}-2}(d-1)^{\frac{2-d}{4}} .
\end{aligned}
$$

So the thermal diffusion constant is

$$
\begin{aligned}
D_{T} & =\frac{w^{2}}{\sigma_{\perp} B^{2} \frac{\partial s}{\partial T} T^{2}} \\
& =\frac{2 \pi T}{B \sqrt{d-1}} .
\end{aligned}
$$

It is easy to verify that the expression for the butterfly velocity remains the same,

$$
\begin{aligned}
v_{x_{1}}^{2} & =\frac{4 \pi^{2} T^{2}}{g_{x x}\left(r_{h}\right) m^{2}} \\
& =\frac{4 \pi T g_{y y}\left(r_{h}\right)}{g_{x_{1} x_{1}}\left(r_{h}\right) g_{y y}^{\prime}\left(r_{h}\right)} \\
& =\frac{8 \pi^{2} T^{2}}{B \sqrt{d-1}} .
\end{aligned}
$$

Then the ratio in this case is

$$
\lambda_{L} D_{T} / v_{B}^{2}=\frac{1}{2} .
$$

When $d$ is odd it is an isotropic solution, and when $B / T^{2} \rightarrow$ $\infty$ the horizon radius $r_{h}^{2} \rightarrow r_{+}^{2}=B / \sqrt{d}$. In this case the near-horizon geometry is just $\mathrm{AdS}_{2} \times R^{d-1}$. It has been proven in Ref. [10] that when the near-horizon geometry is $\mathrm{AdS}_{2} \times R^{d-1}$, the ratio of thermal diffusion $\lambda_{L} D_{T} / v_{B}^{2}=1$. 
[1] S. A. Hartnoll, Nat. Phys. 11, 54 (2015).

[2] S. H. Shenker and D. Stanford, J. High Energy Phys. 03 (2014) 067.

[3] D. A. Roberts, D. Stanford, and L. Susskind, J. High Energy Phys. 03 (2015) 051.

[4] S. H. Shenker and D. Stanford, J. High Energy Phys. 05 (2015) 132.

[5] J. Maldacena, S. H. Shenker, and D. Stanford, J. High Energy Phys. 08 (2016) 106.

[6] K. Jensen, Phys. Rev. Lett. 117, 111601 (2016).

[7] M. Blake, Phys. Rev. Lett. 117, 091601 (2016).

[8] M. Blake, Phys. Rev. D 94, 086014 (2016).

[9] Y. Ling, P. Liu, and J. P. Wu, J. High Energy Phys. 10 (2017) 025.

[10] M. Blake and A. Donos, J. High Energy Phys. 02 (2017) 013.

[11] S. F. Wu, B. Wang, X. H. Ge, and Y. Tian, Phys. Rev. D 97, 106018 (2018).

[12] K. Y. Kim and C. Niu, J. High Energy Phys. 06 (2017) 030.

[13] M. Blake, R. A. Davison, and S. Sachdev, Phys. Rev. D 96, 106008 (2017).

[14] M. Baggioli and W. J. Li, J. High Energy Phys. 07 (2017) 055.

[15] Y. Ling and Z. Y. Xian, J. High Energy Phys. 09 (2017) 003.

[16] D. Giataganas, U. Grsoy, and J. F. Pedraza, Phys. Rev. Lett. 121, 121601 (2018).

[17] H. S. Jeong, Y. Ahn, D. Ahn, C. Niu, W. J. Li, and K. Y. Kim, J. High Energy Phys. 01 (2018) 140.

[18] X. H. Ge, S. J. Sin, Y. Tian, S. F. Wu, and S. Y. Wu, J. High Energy Phys. 01 (2018) 068.

[19] D. Avila, V. Jahnke, and L. Patio, J. High Energy Phys. 09 (2018) 131.

[20] Y. Gu, X. L. Qi, and D. Stanford, J. High Energy Phys. 05 (2017) 125.

[21] Y. Gu, A. Lucas, and X. L. Qi, SciPost Phys. 2, 018 (2017).

[22] Y. Chen, H. Zhai, and P. Zhang, J. High Energy Phys. 07 (2017) 150.

[23] W. Cai, X. H. Ge, and G. H. Yang, J. High Energy Phys. 01 (2018) 076.
[24] H. Guo, Y. Gu, and S. Sachdev, Phys. Rev. B 100, 045140 (2019).

[25] M. Baggioli, B. Goutraux, E. Kiritsis, and W. J. Li, J. High Energy Phys. 03 (2017) 170.

[26] A. A. Patel and S. Sachdev, Proc. Natl. Acad. Sci. U.S.A. 114, 1844 (2017).

[27] R. A. Davison, W. Fu, A. Georges, Y. Gu, K. Jensen, and S. Sachdev, Phys. Rev. B 95, 155131 (2017).

[28] A. Lucas and J. Steinberg, J. High Energy Phys. 10 (2016) 143.

[29] W. J. Li, P. Liu, and J. P. Wu, J. High Energy Phys. 04 (2018) 115.

[30] A. Mokhtari, S. A. Hosseini Mansoori, and K. B. Fadafan, Phys. Lett. B 785, 591 (2018).

[31] E. D’Hoker and P. Kraus, J. High Energy Phys. 10 (2009) 088.

[32] S. Grozdanov, K. Schalm, and V. Scopelliti, Phys. Rev. Lett. 120, 231601 (2018).

[33] M. Blake, H. Lee, and H. Liu, J. High Energy Phys. 10 (2018) 127.

[34] M. Blake, R. A. Davison, S. Grozdanov, and H. Liu, J. High Energy Phys. 10 (2018) 035.

[35] M. Blake, R. A. Davison, and D. Vegh, arXiv:1904.12883.

[36] S. Grozdanov, P. K. Kovtun, A. O. Starinets, and P. Tadi, arXiv:1904.12862.

[37] J. Hernandez and P. Kovtun, J. High Energy Phys. 05 (2017) 001.

[38] S. Grozdanov, D. M. Hofman, and N. Iqbal, Phys. Rev. D 95, 096003 (2017).

[39] I. Papadimitriou, J. High Energy Phys. 08 (2011) 119.

[40] A. Donos, J. P. Gauntlett, and V. Ziogas, J. High Energy Phys. 03 (2018) 056.

[41] A. Donos and J. P. Gauntlett, J. High Energy Phys. 11 (2014) 081.

[42] W. Li, S. Lin, and J. Mei, Phys. Rev. D 98, 114014 (2018).

[43] S. Grozdanov and N. Poovuttikul, J. High Energy Phys. 04 (2019) 141.

[44] D. M. Hofman and N. Iqbal, SciPost Phys. 4, 005 (2018). 